\title{
PROPOSTA DE IMPLEMENTAÇÃO DO BIM NA ACADEMIA
}

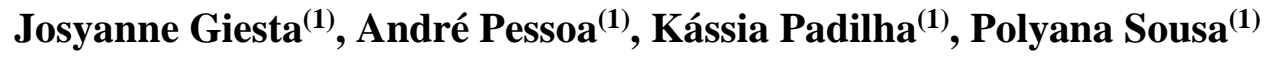 \\ (1) Instituto Federal de Educação, Ciência e Tecnologia do Rio Grande do Norte, Natal
}

\begin{abstract}
Resumo
O desenvolvimento da produtividade é importante para a indústria brasileira da Construção Civil, a qual apresenta o Building Information Modeling (BIM) como uma realidade que ainda é mais evidente com o Decreto Federal ${ }^{\circ} 10.306$, promulgado no ano de 2020 . Nesse cenário, percebe-se que o atual mercado da área de Arquitetura, Engenharia e Construção (AEC), exige profissionais capacitados nessa tecnologia. Sendo premente que a academia se posicione frente à preparação adequada de profissionais BIM. Pensando nessa realidade, o presente trabalho tem como objetivo propor métodos de inserção do BIM na matriz curricular do curso técnico em Edificações na modalidade Subsequente, do Instituto Federal do Rio Grande do Norte (IFRN). O método de pesquisa adotado foi a Design Science Research (DSR), com a proposta de experiência prática em duas disciplinas, Construção Civil 1 e Instalações Elétricas, utilizando o ensino híbrido. Como resultado, foram propostas diretrizes para inserção do BIM utilizando ensino híbrido. As diretrizes propostas mostram que mesmo diante de cursos AEC com disciplinas e ementas já consolidadas, é possível através de metodologias ativas, inserir o BIM nas disciplinas.
\end{abstract}

\section{Introdução}

O Decreto Federal $N^{\circ} 10.306$ de 2 de abril de 2020 [1] define que os profissionais contratados deverão estar habilitados e com experiência ou formação Building Information Modeling (BIM), reforçando a urgência no processo de implementação do BIM nas instituições de ensino. Nesse cenário, é importante destacar que ainda são poucos os estudos que versam sobre esse processo de implementação do paradigma. Pode-se destacar o de [2], que trata do desenvolvimento de um método que pode ser aplicado para auxiliar na inserção de BIM nos currículos de cursos de graduação em Engenharia Civil. E o de [3], que avaliou o currículo do curso de Engenharia Civil da UFRN, utilizando o Método Checcucci. Outro trabalho relevante é [4], que analisou matrizes curriculares de cursos de graduação em Arquitetura e Urbanismo de São Paulo para identificar como estava sendo implantado o BIM. Observa-se que os trabalhos tratam da implementação do BIM em cursos de graduação, ficando uma lacuna no que se refere a cursos técnicos de nível médio. 
Diante desse quadro, o presente artigo tem como objetivo desenvolver uma proposta de implementação do BIM para o curso técnico de nível médio em Edificações. No entanto, diante de um curso que já tem conteúdos e carga horária definidos, é evidente a dificuldade na inserção de conteúdos BIM, sem comprometer os conteúdos técnicos nem aumentar a carga horária. Para tanto, a proposta trabalha com a utilização da metodologia ativa - ensino híbrido, propondo a atualização do curso de Edificações concatenando as metodologias tradicionais de ensino com novas metodologias.

\section{Inserção do BIM na academia}

\subsection{Importância do BIM no Ensino}

O BIM está se expandindo de maneira vasta em todo o mundo pela sua capacidade de dimensionar, planejar, coordenar e recuperar informações a qualquer momento, bem como, verificar interferências, testar alternativas de projeto, ensaiar o comportamento do modelo sob o uso de diversos componentes [5]. Seus resultados positivos, o impulsionam para ser o principal elemento na revolução do novo cenário de trabalho na construção civil mundial. No Brasil, apesar das dificuldades e do atraso comparado a outros países, já foi concedido pelo governo federal, que o uso do BIM seja estabelecido na execução direta e indireta de obras e serviços de engenharia realizada pelos órgãos e pelas entidades da administração pública federal [1]. Com esta perspectiva, se torna nítido a importância desse paradigma, remetendo a uma demanda por profissionais habilitados e consequentemente pelo ensino do BIM nos cursos da área de Arquitetura, Engenharia e Construção (AEC).

Diante desse cenário, a academia vem promovendo pesquisas sobre BIM, no entanto, a maioria dessas pesquisas versam sobre Implantação do paradigma, segundo os resultados da Revisão Sistemática da Literatura realizada por [6], dos 10 temas propostos, Ensino, Colaboração e Manutenção são os menos pesquisados.

É inevitável o incremento do BIM na academia, objetivando capacitar os futuros profissionais, fortalecendo uma proposta de graduandos bem instruídos e atualizados para o mercado de trabalho. Para acompanhar esse propósito as instituições de ensino têm utilizado duas modalidades de ensino, com disciplinas isoladas com conteúdos BIM e a pulverização dos conteúdos BIM por várias disciplinas do currículo. Nesse sentido, [7] defendem a adoção do BIM integrada às diversas disciplinas da matriz curricular, em contraponto a implantação pontual do paradigma.

Sobre estratégias de introdução do BIM, [8] apresentam a existência de três tipos: Colaboração intracursos, Colaboração interdisciplinar e Colaboração à distância. Na colaboração intracursos estão experiências em que o BIM é introduzido em apenas um curso. Na colaboração interdisciplinar temos exemplos de escolas que integram disciplinas de dois ou três cursos distintos, enquanto na colaboração à distância tem-se a simulação de uma verdadeira colaboração com alunos de duas ou mais escolas distantes.

\subsection{Metodologias ativas no ensino do BIM}

As instituições de ensino têm um papel importante que é fazer com que a próxima geração de profissionais entendam o BIM como uma tecnologia que suporta o trabalho em colaboração [9]. Vale ressaltar que apesar do desenvolvimento das metodologias inovadoras de ensino, o modelo tradicional ainda é amplamente aplicado nas instituições de ensino [10]. Nessa conduta, 
pesquisas apontam que o método tradicional traz desvantagem para o processo de conhecimentos que podem ser filtrados para os estudantes do meio acadêmico. Com esse modelo, tudo que é dado como informação na interação do professor para com o aluno demonstra um sistema de memorização, ao invés de expansão do conhecimento, interferindo também no desenvolvimento da reflexão e senso crítico [10]. Tendo em vista que um profissional precisa em sua formação ser contextualizado para competir em um mercado de trabalho que demanda cada vez mais qualidade, produtividade, competitividade, entre outros atributos.

Os novos métodos de ensino acompanham os avanços tecnológicos e as novas formas de trabalho. Os educadores devem usar essas novas tecnologias a favor de novas propostas metodológicas para o ensino-aprendizagem. Essas novas propostas por sua vez, devem atender às mudanças dos conteúdos e do perfil do aluno, sem perder a qualidade e a base teórica [10]. Portanto, a tecnologia deve ajudar as novas metodologias a atingir os objetivos propostos.

As metodologias ativas são maneiras de valorizar o aluno para torná-lo um excelente profissional, com mais criatividade, raciocínio lógico e crítico, despertando assim a independência dos estudantes, pois as academias têm um papel importante, que é o de aproximar essa nova geração do entendimento de tecnologias facilitadoras para o trabalho profissional, como o BIM [9].

Sobre metodologias de aprendizagem ativa, [11] apontam que os ambientes híbridos permitem aos estudantes expandirem seus conhecimentos sobre BIM. O ensino híbrido, envolve o estudo presencial e o virtual, expondo o aluno às tecnologias do ensino online. $\mathrm{O}$ acesso aos conteúdos pode ser realizado em casa e as discussões em sala de aula, promovendo assim a reflexão e a visão crítica do aluno.

Um dos diversos meios utilizados para desenvolver as metodologias ativas BIM no ensino, é o processo de Sala de Aula Invertida. Nela, já são previamente disponibilizados aos alunos, o conteúdo, para em aula acontecerem debates entre professores e alunos, consequentemente alterando a lógica da organização do ensino presencial, pois os alunos precisam estudar o conteúdo antes da aula. Proporcionando diversos pontos de vista, a aula se torna mais dinâmica, promovendo mais atividades em grupos e aguçando mais o conhecimento [10].

Outro exemplo de ensino híbrido é o Laboratório Rotacional, que segundo [12] começa com a sala de aula tradicional, e em seguida adiciona uma rotação para um computador ou laboratório de ensino. Ainda de acordo com a autora, os Laboratórios Rotacionais, frequentemente aumentam a eficiência operacional e facilitam o aprendizado personalizado, mas não substituem o foco nas ações convencionais que ocorrem em sala de aula. Nesse modelo, os alunos que forem direcionados ao laboratório trabalharão nos computadores, de forma individual e autônoma, para cumprir os objetivos fixados pelo professor que estará, com outra parte da turma, realizando sua aula.

\section{Curso Técnico em Edificações}

O curso técnico de nível médio em Edificações, na forma subsequente, tem duração de quatro semestres e se caracteriza como uma modalidade para alunos que já concluíram o ensino médio. Segundo o projeto pedagógico do curso o perfil do egresso deve constar de habilidades para o desempenho de atividades voltadas a área profissional da Construção Civil, compreendendo atividades de planejamento, projeto, acompanhamento e orientação técnica à execução e à 
manutenção de obras civis, como edifícios, aeroportos, rodovias, ferrovias, portos, usinas, barragens e vias navegáveis, abrangendo a utilização de técnicas e processos construtivos em escritórios, execução de obras e prestação de serviços [13].

O curso possui carga horária (C.H.) total de $1.700 \mathrm{~h}$, sendo $1.200 \mathrm{~h}$ reservadas para as 15 disciplinas. Está organizado em três núcleos (Figura 1): a) Núcleo Fundamental (disciplinas de revisão do ensino médio); b) Núcleo Articulador (disciplinas de base científica e tecnológica comuns aos eixos tecnológicos e disciplinas técnicas de articulação e integração); e c) Núcleo Tecnológico (disciplinas técnicas do curso, não contempladas no Núcleo Articulador).

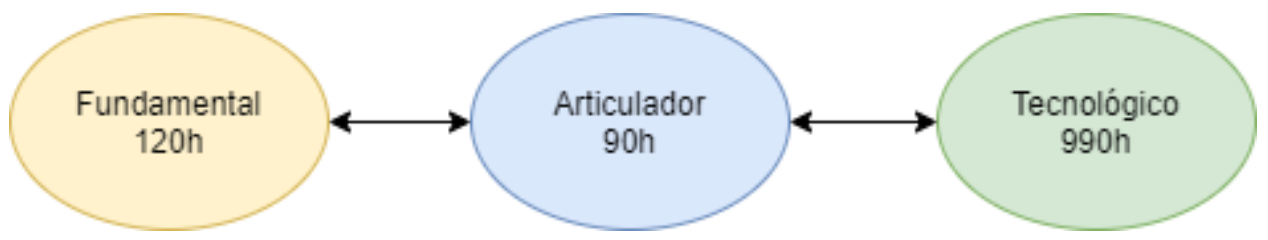

Figura 1: Núcleos do curso de Edificações e suas cargas horárias (Adaptado de [13]).

\section{Metodologia}

Da perspectiva de sua natureza trata-se de uma pesquisa aplicada pois pretende gerar conhecimentos para aplicação prática, direcionados à solução de problemas específicos.

O método aplicado na pesquisa foi o Design Science Research (DSR), com tipo de artefato Método (Figura 2). A escolha pela DSR deu-se pelo fato de que se trata de um método que busca a partir do entendimento do problema, construir e avaliar artefatos que permitam transformar situações, alterando suas condições para estados melhores ou desejáveis [14].

Do ponto de vista teórico, são poucos os estudos na área de ensino BIM, e quando existem são mais direcionados aos cursos de graduação em Engenharia Civil e Arquitetura e Urbanismo, existindo ainda uma lacuna em termos de pesquisas voltadas para cursos técnicos.

Do ponto de vista prático, tem-se a necessidade de inserir conteúdos BIM em uma matriz curricular já definida, sem retirar conteúdos técnicos nem aumentar a C.H. dos componentes curriculares.

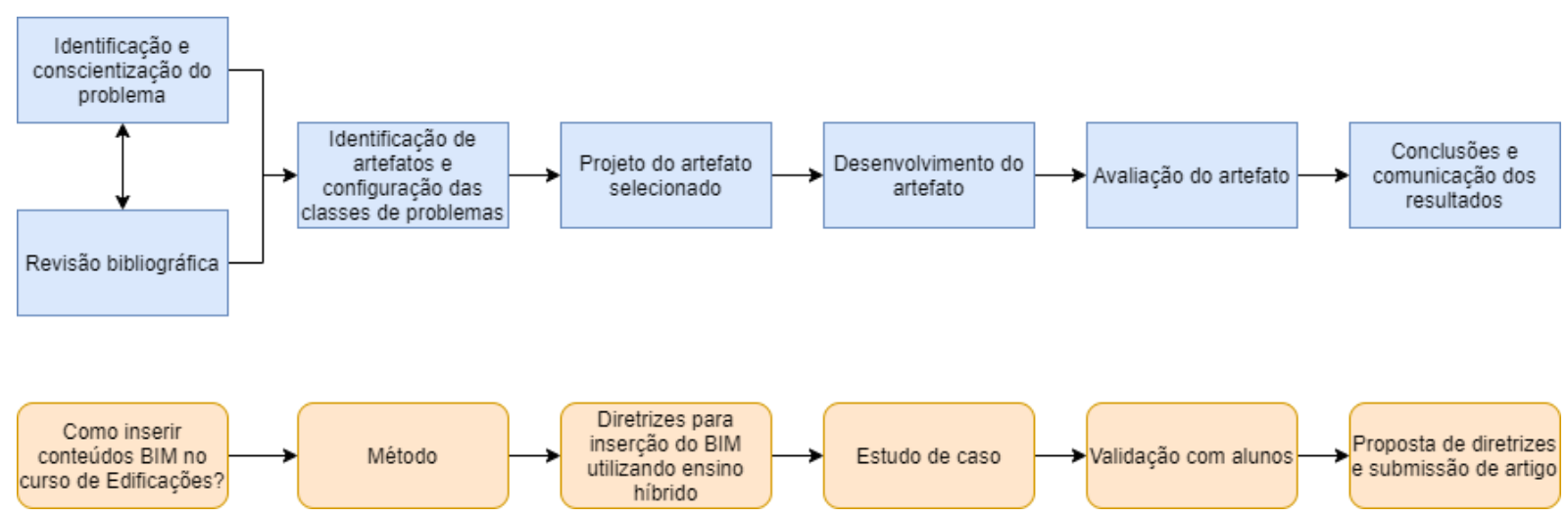

Figura 2: Etapas da Design Science Research (Adaptado de [14]). 


\section{Resultados e discussão}

\subsection{Identificação e conscientização do problema}

A revisão bibliográfica realizada indicou o aumento do interesse na implementação do BIM, demonstrando a necessidade e procura de profissionais habilitados e com competências BIM, por parte do mercado da área de AEC. Aliado a isto tem-se os decretos federais dos últimos anos, que vêm cada vez mais sinalizando o estabelecimento da obrigatoriedade do BIM para determinadas áreas. Diante desse cenário, a academia tem se posicionado, com o surgimento de pesquisas que objetivam a inserção do BIM na matriz curricular, no entanto, as mesmas, tratam em sua maioria dos cursos de graduação em Arquitetura e Urbanismo e Engenharia Civil.

O curso técnico de nível médio em Edificações tem sido pouco contemplado em termos de pesquisas que versem sobre a atualização de seu currículo, para contemplar a inserção de conteúdos BIM. Deste modo, identifica-se o seguinte problema: Como inserir conteúdos BIM no curso de Edificações?

\subsection{Identificação de artefatos e classe de problemas}

Diante da identificação do problema e ciente do contexto de muitos conteúdos BIM a serem inseridos para a formação adequada do profissional, em contraponto a necessidade de manter os conteúdos técnicos existentes, bem como da certeza de que a matriz curricular apresentada no projeto pedagógico do curso de Edificações [13] não contempla horas disponíveis para essa inserção, percebeu-se a necessidade de um artefato que corresponda a um conjunto de passos necessários para desempenhar determinada tarefa, que no caso é o artefato Método.

\subsection{Projeto do artefato}

Com base em [15] identificou-se que os conteúdos BIM podem ser inseridos em praticamente todas as disciplinas do Núcleo Tecnológico da matriz curricular, sendo permitido desde o segundo semestre do curso. Essa possibilidade do BIM de forma pulverizada por várias disciplinas do curso favorece a colaboração entre as disciplinas e com os estudantes de vários semestres do curso, o que caracteriza segundo [8] um modelo de Colaboração intracursos.

Diante desse contexto, a proposta de projeto para o artefato foi a inserção dos conteúdos BIM utilizando o ensino híbrido, entre os quais Laboratório Rotacional e Sala de Aula Invertida (Figura 3).

\begin{tabular}{|c|c|c|c|c|}
\hline Disciplina & Conteúdos BIM & Momentos de inserção & Ensino Híbrido & Local \\
\hline $\mathrm{CC} 1$ & $\begin{array}{c}\text { Modelagem 3D } \\
\text { Extração de } \\
\text { quantittivos através de } \\
\text { tabelas }\end{array}$ & $\begin{array}{c}\text { Aula cálculo de } \\
\text { volume de concreto de } \\
\text { fundações do tipo } \\
\text { sapatas }\end{array}$ & $\begin{array}{c}\text { Laboratório } \\
\text { Rotacional com vídeo } \\
\text { explicativo da } \\
\text { utilização do software }\end{array}$ & $\begin{array}{l}\text { Laboratório } \\
\text { GIP e Sala de } \\
\text { aula tradicional }\end{array}$ \\
\hline IE & $\begin{array}{l}\text { Modelagem 3D } \\
\text { Verificação de } \\
\text { interferência }\end{array}$ & $\begin{array}{l}\text { Aula de lançamento } \\
\text { dos pontos e } \\
\text { lançamento do } \\
\text { eletrodutos }\end{array}$ & $\begin{array}{c}\text { Sala de Aula Invertida } \\
\text { com vídeo explicativo } \\
\text { da utilização do } \\
\text { software }\end{array}$ & $\begin{array}{l}\text { Laboratório } \\
\text { GIP }\end{array}$ \\
\hline
\end{tabular}

Figura 3: Detalhamento das unidades de análise. 
Dentre as disciplinas existentes no currículo do curso, optou-se como unidade de análise as disciplinas de Construção Civil 1 - CC1 (120 h/a) e Instalações Elétricas - IE (80 h/a), pela possibilidade de abordar conteúdos BIM diferentes, bem como pela aceitação por parte dos docentes das disciplinas. O software BIM escolhido foi o Autodesk Revit, versão 2020 em razão de ser um software do conhecimento dos autores, bem como de ser o existente no laboratório do Grupo de Estudos e Pesquisa em Integração de Projetos (GIP).

\subsection{Desenvolvimento do artefato}

Nessa etapa, com base nas diretrizes desenvolvidas (Figura 4), deu-se início a experimentação na unidade de estudo Construção Civil 1, a turma composta por 18 alunos encontra-se no segundo período do curso de Edificações, modalidade subsequente do IFRN - Campus Natal Central. Antes de aplicar o estudo de caso, houve todo um planejamento diante da quantidade de alunos e do suporte tecnológico existente, a saber sete computadores, localizados no GIP.

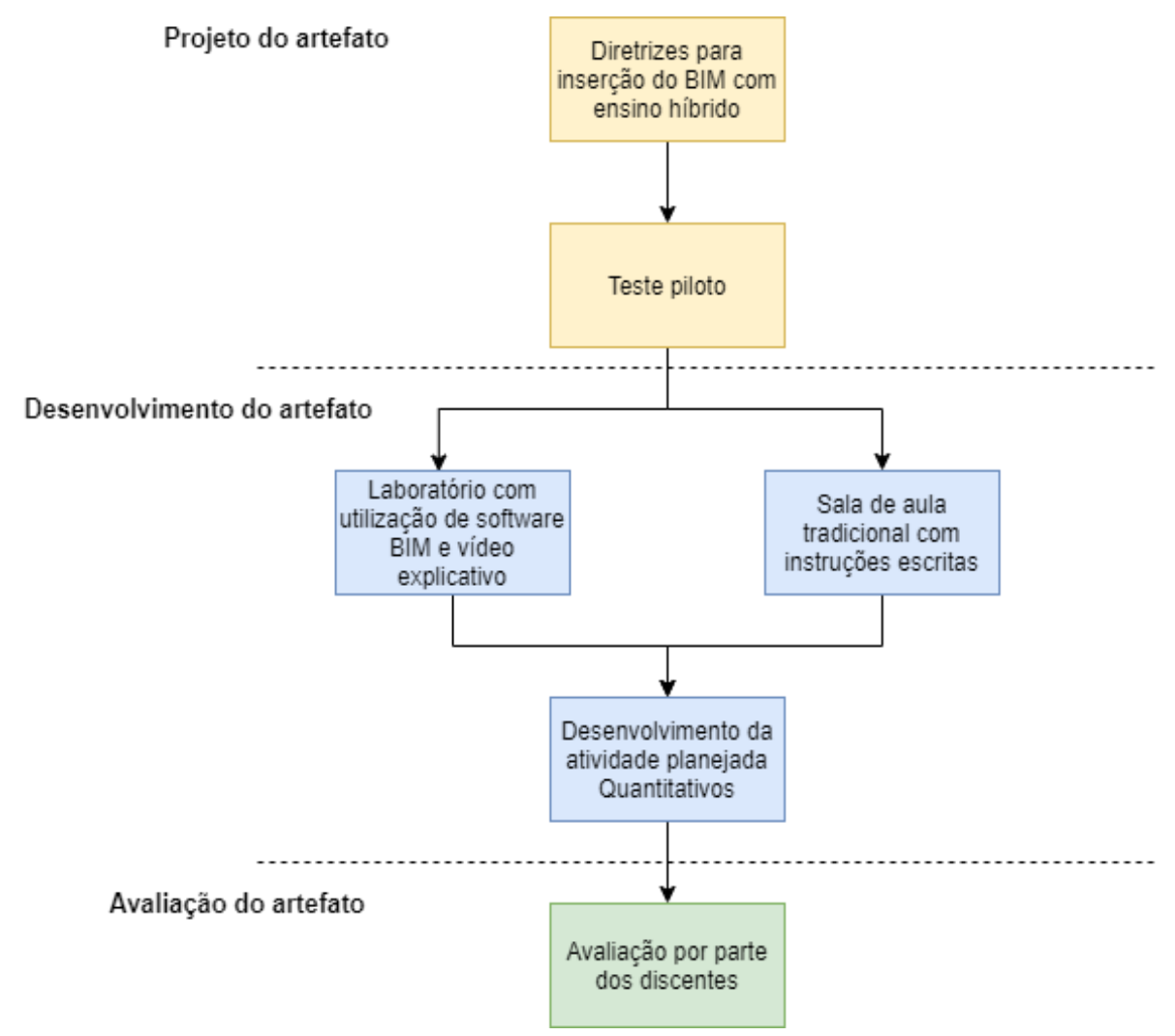

Figura 4: Desenvolvimento do artefato.

Objetivando a utilização do ensino híbrido - Laboratório Rotacional, os alunos foram divididos em dois grupos, Grupo A - sala tradicional e Grupo B - GIP. Para o Grupo A foi desenvolvido um procedimento escrito com instruções para a extração dos quantitativos do volume de concreto com o uso de calculadora. Enquanto, que para o Grupo B foi desenvolvido um vídeo explicativo gravado por meio de um software que captura ações da tela, descrevendo um passo a passo sobre como extrair os quantitativos utilizando tabelas através de software BIM.

O projeto proposto para o estudo de caso era composto por dezesseis sapatas (Figura 5), distribuídas em quatro grupos com as seguintes dimensões $(2,00 \times 2,70 ; 2,30 \times 2,60 ; 2,10 \times 2,20$ e 
1,60x1,70). Com base no teste piloto realizado anteriormente, concluiu-se por determinar um tempo para a execução da experiência de 90 minutos.

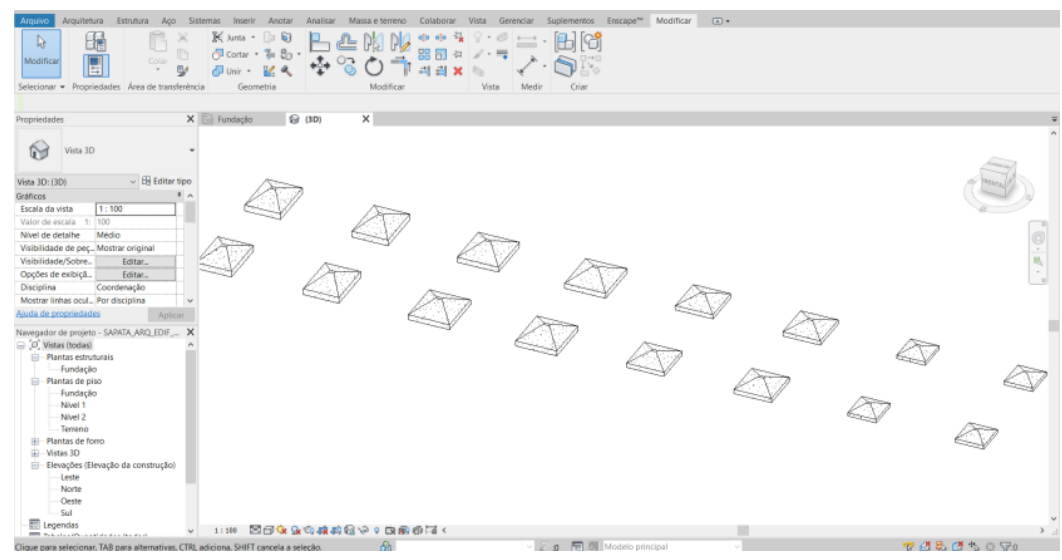

Figura 5: Projeto de fundações do tipo sapatas.

Com os materiais prontos e as formas de aplicação devidamente planejadas, deu-se início ao estudo de caso na sala de aula tradicional, no horário da aula de Construção Civil 1. Na sequência, o outro grupo de alunos se deslocou para o laboratório GIP, onde os computadores já se encontravam ligados e devidamente identificados. Devido a quantidade limitada de computadores, o Grupo B foi dividido em duas etapas.

\subsection{Avaliação do artefato}

A avaliação do artefato se deu com a aplicação de um questionário, logo após a conclusão da atividade proposta. No caso dos alunos do Grupo B é relevante apontar que eles classificaram o vídeo explicativo como sendo de grande valia para a aprendizagem do software BIM, indicando ainda que sentiram facilidade no manuseio do software, mesmo metade deles tendo afirmado que desconheciam o software antes da experiência. Além disso, quase que a totalidade dos alunos concordaram que seria viável a utilização de vídeo para o ensino do software, os alunos ressaltaram ainda o desejo de que o software seja utilizado com maior frequência durante as aulas.

Os alunos, de modo geral, elencaram como principal vantagem da utilização do software BIM, a velocidade de resolução dos problemas, seguida dos quantitativos práticos, redução de erros e visualização (Figura 6).

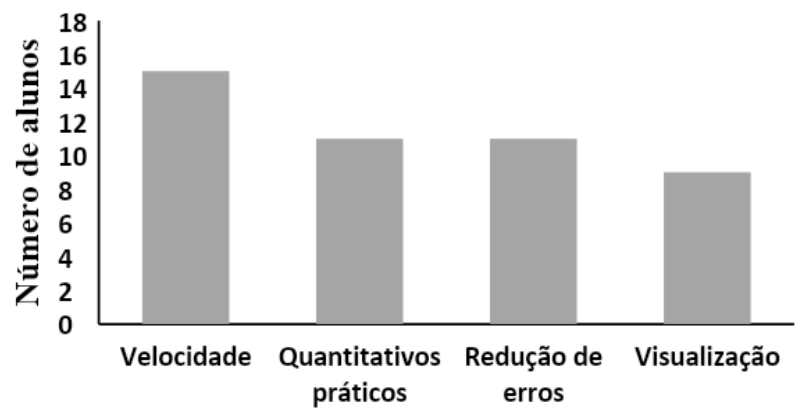

Figura 6: Vantagens da utilização do software BIM. 
Como sugestão para otimização do processo de ensino, utilizando o software BIM, os alunos indicaram, a solução de dúvidas via chat, a otimização dos vídeos e o acesso aos arquivos utilizados no GIP.

\subsection{Conclusões e comunicação dos resultados}

Com relação ao tempo necessário para a conclusão da atividade proposta, foi identificado uma grande distorção entre os Grupos A e B (Figura 7). O Grupo A demandou maior tempo no cálculo do volume de concreto proposto, em função de dificuldades em solucionar cálculos e interpretar instruções escritas que apresentam fórmulas, sem a presença de um professor orientador. Em contraponto, os alunos do Grupo B demandaram menor tempo na resolução, devido as instruções por vídeo e a utilização de software BIM.

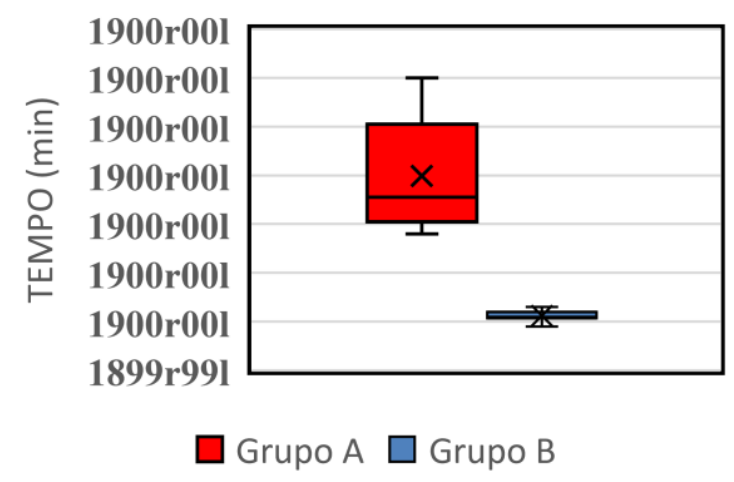

Figura 7: Duração da atividade proposta na disciplina de Construção Civil 1.

A diferença no tempo de resolução, indicou uma maior dificuldade no processo, o que foi refletido na quantidade de acertos no cálculo do volume de concreto entre os dois grupos (Figura 8). É possível perceber que, o número de acertos foi inversamente proporcional ao tempo de resolução.

Mesmo os alunos do Grupo A tendo demonstrado estarem seguros de suas respostas com relação aos cálculos, nenhum conseguiu chegar ao resultado esperado, indicando a necessidade da presença de um professor orientador. No entanto, o Grupo B apresentou maior sucesso no cálculo do volume de concreto, chegando a obter um percentual de $80 \%$ de acerto.

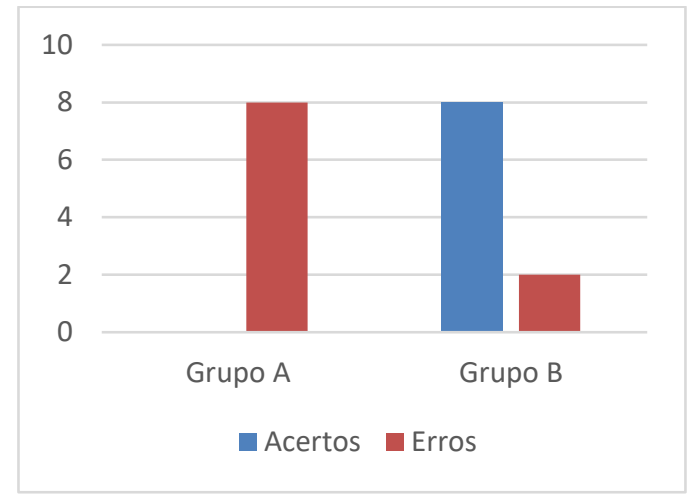

Figura 8: Grau de acerto no cálculo do volume de concreto. 
Diante do exposto identifica-se que a proposta de inserção de conteúdos BIM, através do ensino híbrido - Laboratório Rotacional, com a utilização de tecnologia - vídeo explicativo apresentouse como uma estratégia positiva, tendo alcançado melhores resultados em termos de menor carga horária necessária para o ensino do conteúdo, maior precisão nos cálculos, bem como de grande aceitabilidade por parte dos alunos envolvidos. Como forma de comunicação dos resultados positivos da experimentação, foi realizada a elaboração desse artigo científico, submetido ao evento ptBIM 2020.

\section{Considerações Finais}

Este trabalho objetivou o desenvolvimento de uma proposta de inserção do BIM no curso técnico de nível médio em Edificações, para tanto as diretrizes criadas priorizaram a utilização do ensino híbrido, de forma a permitir a inserção de conteúdos BIM sem a necessidade de incrementos de carga horária ou substituição dos conteúdos existentes.

Com relação as principais contribuições do trabalho, pode-se destacar do ponto de vista teórico o desenvolvimento do artigo, com as diretrizes para a inserção do BIM no curso de Edificações, utilizando ensino híbrido. Com relação ao ponto de vista prático, a experimentação do ensino do BIM na disciplina de Construção Civil 1, utilizando o Laboratório Rotacional se caracterizou como uma excelente opção a ser aplicada.

Há que se apontar que os resultados obtidos corroboram com outras pesquisas, que indicam que os alunos dessa nova geração, nasceram na tecnologia, e portanto, apresentam um grande potencial de aprendizagem quando se trata de utilização de softwares, bem como maior facilidade para aprendizagem, quando colocados na posição de protagonistas.

Como limitações da pesquisa pode-se apontar a proposta de apenas duas modalidades de ensino híbrido, bem como de somente duas disciplinas do curso. Essas limitações indicam como sugestões para trabalhos futuros a utilização de outros modelos de ensino híbrido. Bem como, que a experimentação seja replicada com outras disciplinas do curso de Edificações, de forma a contribuir com o processo de inserção do Building Information Modeling na academia.

\section{Referências}

[1] BRASIL. "Decreto n ${ }^{0} 10.306$, de 02 de abril de 2020". Dispõe sobre a utilização do Building Information Modelling - BIM ou Modelagem da Informação da Construção na execução direta ou indireta de obras e serviços de engenharia, realizada pelos órgãos e pelas entidades da administração pública federal, no âmbito da Estratégia Nacional de Disseminação do Building Information Modelling - Estratégia BIM BR. Diário Oficial da República Federativa do Brasil, Brasília, n. 65, Seção 1, p. 5-7, abr. 2020.

[2] E. S. Checcucci. "Ensino-aprendizagem de BIM nos cursos de graduação em Engenharia Civil e o papel da Expressão Gráfica neste contexto". 2014. 17 f. il. Tese (Doutorado em Difusão do Conhecimento) - Faculdade de Educação, Universidade Federal da Bahia, Salvador, 2014.

[3] W. E. F. Lima; L. A. P. Melo; R. S. S. Melo; J. P. Giesta. Interfaces entre o curso de engenharia civil da UFRN e BIM: Uma análise da matriz curricular In: Simpósio Brasileiro de Tecnologia da Informação e Comunicação na Construção, 2., 2019, Campinas, SP. 
Anais[...] Porto Alegre: ANTAC, 2019. Disponível em: https://antaceventos.net.br/index.php/sbtic/sbtic2019/paper/view/140. Acesso em: 10 jul. 2020.

[4] L. A. Santos. "Building information modeling no ensino de arquitetura e urbanismo: Percepção e disseminação do BIM nas Instituições de Ensino Superior do Estado de São Paulo". 2017. 138 f. Dissertação (Mestrado em arquitetura e urbanismo) - Universidade São Judas Tadeu, São Paulo, 2017.

[5] A. Roux; M. Addor; M. D. de A. Castanho; H. Cambiaghi; J. P. M. Delatorre; E. S. Nardelli; A. L. de Oliveira. (2019). Colocando o "I" no BIM. arq.Urb, (4), 104-115.

[6] J. F. L. Coelho. "Estudo empírico para proposta de diretrizes para implantação do BIM em pequenas e médias empresas no Brasil". 2017. 101 f. Dissertação de Mestrado em Engenharia Civil. Pontifícia Universidade Católica do Rio de Janeiro. Rio de Janeiro, 2017.

[7] E. S. Checcucci; A. P. C. Pereira; A. L. de Amorim. Modelagem da Informação da Construção (BIM) no Ensino de Arquitetura. Proceedings of the XVII Conference of the Iberoamerican Society of Digital Graphics: Knowledge-based Design. Anais... In: SIGRADI 2013. Valparaíso, Chile: 2013.

[8] M. B. Barison; E. T. Santos. Estratégias de ensino BIM: uma visão geral das abordagens atuais. 2010. Disponível em: http://www.uel.br/pessoal/barison/Artigos_Tese/p288p.pdf. Acesso em: 10 jul. 2020.

[9] M. B. Barison; E. T. Santos. Tendências Atuais para o Ensino de BIM. In: Encontro Nacional de Tecnologia de Informação e Comunicação na Construção, 5., 2011, Salvador. Tendências Atuais para o Ensino de BIM. Salvador: SBTIC, 2011. p. 1 - 14.

[10] D. V. dos Santos. "Ensino de BIM na disciplina Modelagem da Informação e da Construção (MIC) por meio das Metodologias Ativas". 2017. 96 f. TCC (Graduação) - Curso de Engenharia Civil, Centro de Tecnologia, Universidade Federal do Ceará (UFC), Fortaleza, 2017. Cap. 5. Disponível em: http://www.repositorio.ufc.br/handle/riufc/28845. Acesso em: 29 fev. 2020.

[11] A. R. M. Cuperschmid; C. M. Castriotto. Teaching BIM modeling in the architecture course: using a Blended Learning Strategy, p. 942-947. São Paulo: Blucher, 2018. ISSN 2318-6968, DOI 10.5151/sigradi2018-1245.

[12]L. Bacich. "Implicações da organização da atividade didática com uso de tecnologias digitais na formação de conceitos em uma proposta de Ensino Híbrido". 2016. 317 f. Tese (Doutorado em Psicologia) - Universidade de São Paulo, São Paulo, 2016.

[13] Instituto Federal de Educação, Ciência e Tecnologia do Rio Grande do Norte (IFRN). "Projeto Pedagógico do Curso Técnico de Nível Médio em Edificações". Natal: IFRN, 2011.

[14] A. Dresch; D. P. Lacerda; J. A. V. Antunes Júnior. Design Science Research: método de pesquisa para avanço da ciência e tecnologia. Porto Alegre: Bookman, 2015. 181 p.

[15] J. P. Giesta; G. L. B. B. de Menezes; A. Costa Neto. Integração do BIM no currículo do curso de Edificações. In: Simpósio Brasileiro de Tecnologia da Informação e Comunicação na Construção, 1, 2017, Fortaleza, CE. Anais... Disponível em: https://sbtic.com.br/anais/files/2017/paper_64.pdf. Acesso em: 10 jul. 2020. 